\title{
Angioplastia primaria en el infarto agudo de miocardio: ¿es beneficioso el condicionamiento isquémico remoto?
}

\section{Resumen}

El paradigma de la reapertura precoz de la arteria ocluida ha representado un hito en el tratamiento del infarto agudo de miocardio y su aplicación universal en la práctica cardiológica se tradujo en una reducción significativa de la magnitud del daño miocárdico y de la mortalidad por esta causa.

Sin embargo, la oclusión coronaria aguda continúa siendo una causa prevalente de muerte y discapacidad de origen cardíaco, por lo cual persiste la búsqueda de nuevos procedimientos, fármacos o estrategias que contribuyan a una mayor preservación del tejido miocárdico en este contexto.

El precondicionamiento isquémico cardíaco, en sus diferentes modalidades, a pesar de haber sido objeto de diversos estudios tanto a nivel clínico como experimental, no forma parte de la práctica habitual en los laboratorios de cateterismo cardíaco, servicios de cardiología crítica o unidades de emergencia móvil donde se lleva a cabo la reperfusión miocárdica.

En esta controversia, los autores presentan argumentos a favor y en contra de la utilización del precondicionamiento isquémico remoto como adjunto a la angioplastia primaria en un escenario clínico concreto, brindando al lector el estado de la evidencia sobre un procedimiento cuyo margen de utilidad aguarda aún una clara definición.

Palabras clave: $\quad$ INFARTO DEL MIOCARDIO CON ELEVACIÓN DEL ST

REPERFUSIÓN MIOCÁRDICA

DAÑO POR REPERFUSIÓN

CONDICIONAMIENTO ISQUÉMICO REMOTO

\section{Primary angioplasty in acute myocardial infarction: is remote ischemic conditioning beneficial?}

\section{Summary}

Early reopening of the occluded artery paradigm has represented a milestone in the treatment of acute myocardial infarction, and its universal application in cardiological practice lead to a significant reduction in the extension of myocardial injury and mortality from this cause.

However, acute coronary occlusion is still a prevalent cause of death and disability; therefore, a lively interest in identificating new procedures, drugs or strategies that contribute to a greater preservation of myocardial tissue persists in this context.

Cardiac ischemic preconditioning in its various forms, despite having been object of several studies, both at clinical and experimental levels, is not part of the usual practice of catheterization laboratories, critical cardiology services or mobile emergency units, where myocardial reperfusion is carried out.

In this controversy, the authors present arguments for and against the use of remote cardiac ischemic preconditioning as an adjunct to primary angioplasty in a particular clinical stage, providing the reader the status of evidence about a procedure whose profit margin still awaits a clear definition.

Key words: $\quad$ ST ELEVATION MYOCARDIAL INFARCTION

MYOCARDIAL REPERFUSION

REPERFUSION INJURY

REMOTE ISCHEMIC CONDITIONING

\section{Angioplastia primária no infarto agudo do miocárdio: o condicionamento isquêmico remoto é benéfico?}

\section{Resumo}

O paradigma da reabertura precoce da artéria ocluída representou um marco histórico no tratamento do infarto agudo do miocárdio, e sua aplicação universal na prática cardiológica resultou em uma redução significativa da extensão da lesão miocárdica e da mortalidade por essa causa.

No entanto, a oclusão coronariana aguda continua sendo uma causa prevalente de morte e incapacidade de origem cardíaca, persistindo, portanto, a busca de novos métodos, drogas ou estratégias que contribuam para uma maior preservação do tecido miocárdico nesse contexto.

Pré-condicionamento cardíaco isquêmico, em suas diversas modalidades, que apesar de ter sido objeto de vários estudos, tanto clínicos como experimentais, não faz parte da prática usual no laboratório de cateterismo cardíaco, nos serviços de cardiologia crítica ou nas unidades móveis de emergência, onde a reperfusão miocárdica é realizada. 
Nesta controvérsia, os autores apresentam argumentos a favor e contra o uso do pré-condicionamento isquêmico remoto como adjuvante da angioplastia primária em um ambiente clínico específico, proporcionando ao leitor com o estado da evidência em um procedimento cuja margem de utilidade ainda aguarda uma definição clara.

Palavras chave: $\quad$ INFARTO DO MIOCÁRDIO CON SUPRADESNÍVEL DO SEGMENTO ST REPERFUSÂO MIOCÁRDICA TRAUMATISMO POR REPERFUSÂO CONDICIONAMENTO ISQUÊMICO REMOTO

Viñeta clínica

Paciente de sexo masculino de 46 años, residente en Montevideo. Madre viva, portadora de diabetes mellitus tipo 2. Padre vivo, hipertenso y exfumador. Antecedentes personales de dislipemia, estrés y tabaquismo intenso de larga data. Practica ejercicio moderado regularmente.

Sin sintomatología cardiovascular previa, presenta en reposo dolor retroesternal opresivo, moderadamente intenso, irradiado a maxilar inferior, acompañado de sudoración. Es visto por una unidad de emergencia móvil a los 30 minutos de evolución ininterrumpida del dolor.

$\mathrm{Al}$ examen: índice de masa corporal $23,8 \mathrm{~kg} / \mathrm{m}^{2}$, lúcido; piel húmeda y normocoloreada. Ritmo regular de 106 cpm. Ruidos cardíacos normales, sin R3 ni soplos. Pulsos llenos; presión arterial: 115/70 mmHg. Sin signos congestivos sistémicos. Ventilación conservada a $20 \mathrm{rpm}$, con algunos estertores húmedos en ambas bases. Saturometría: 96\%. Abdomen y miembros inferiores sin particularidades.

Electrocardiograma: ritmo sinusal de $110 \mathrm{cpm}$. Onda P y P-R normales. QRS 0,10". Supradesnivel del segmento $\mathrm{S}-\mathrm{T}$ de hasta $2,5 \mathrm{~mm}$ de $\mathrm{V}_{1}$ a $\mathrm{V}_{5}$ con imagen especular en derivaciones inferiores.

Alivio del dolor con morfina intravenosa. Se administran $325 \mathrm{mg}$ de ácido acetilsalicílico y se traslada a un centro con servicio de hemodinamia activo al momento del arribo, a los 55 minutos de inicio de los síntomas y sin cambios hemodinámicos.

Cineangiocoronariografía: tronco de coronaria izquierda sin lesiones. Arteria descendente anterior (ADA) con oclusión total de aspecto trombótico sobre placa no obstructiva previo al origen de la primera asistencia domiciliaria integral y flujo distal TIMI 0. Arteria circunfleja sin lesiones. Arteria carótida derecha dominante, con irregularidades no significativas en tercio proximal.

Se realiza angioplastia primaria sobre la lesión oclusiva con implante de stent farmacoactivo. ADA con buen lecho distal, extenso territorio y flujo TIMI 3. Estabilidad hemodinámica; pasa a unidad cardiológica.

\section{La utilización del condicionamiento isquémico remoto en el paciente que se presenta podría reducir la magnitud de la necrosis miocárdica o sus consecuencias clínicas}

\section{Dr. Marcelo Espiñeira}

\section{Introducción}

La enfermedad cardiovascular es la primera causa de muerte en el mundo desarrollado, siendo la cardiopatía isquémica aguda la principal causa de morbilidad y mortalidad ${ }^{(1)}$. Si bien la angioplastia primaria es la estrategia más efectiva para reducir el tamaño del infarto (TI), preservar la función ventri- cular y mejorar el pronóstico clínico en pacientes con infarto con supradesnivel del ST (STEMI) ${ }^{(2)}$, la reperfusión en sí misma supone un daño al miocardio, conocido como daño por isquemia-reperfusión (DIR), contribuyendo en 40\%-50\% en el $\mathrm{TI}^{(3)}$, de lo que surge la necesidad de generar estrategias de cardioprotección.

Hospital y Cooperativa Médica de Rivera, Uruguay.

Correspondencia: Artigas M 291, S 3, El Pinar, Canelones, Uruguay.

Correo electrónico: marceloespineira@gmail.com

$\mathrm{El}$ autor declara no tener conflictos de intereses.

Recibido Abr 15, 2019; aceptado Jun 8, 2019. 
El condicionamiento isquémico remoto (CIR) es una estrategia no invasiva, de fácil aplicación, en la cual episodios transitorios y reversibles de isquemia/reperfusión aplicados en un miembro, tejido $\mathrm{u}$ órgano confieren protección frente al daño por isquemia/reperfusión en órganos distantes. El CIR puede ser realizado durante el curso de la isquemia: percondicionamiento (per-CIR), o inmediatamente después de restablecido el flujo (pos-CIR).

En el caso clínico planteado, por tratarse de un paciente joven, con un extenso territorio miocárdico comprometido (supradesnivel del ST anterolateral) y que llega a un centro de hemodinamia activo a los 55 minutos de iniciada la sintomatología, la utilización del CIR podría reducir la magnitud de la necrosis miocárdica y sus consecuencias clínicas. Fundamentamos esta afirmación en base a resultados de ensayos clínicos, metaanálisis y análisis post-hoc que a continuación se analizan.

Botker y colaboradores en el CONDI trial(4), de per-CIR (cuatro ciclos de 5 minutos de insuflación-desinsuflación del esfingomanómetro en el miembro superior) realizado durante el traslado en ambulancia para procedimiento cardiológico intervencionista (PCI), resultó en un incremento de $36 \%$ de miocardio salvado en relación con el área en riesgo medida por imágenes de perfusión miocárdica. Este estudio analizó 142 pacientes con STEMI.

Sloth y colaboradores ${ }^{(5)}$ demostraron en un trabajo realizado en 251 pacientes con STEMI que el per-CIR (cuatro ciclos de 5 minutos de insuflación-desinsuflación del esfingomanómetro en el miembro superior) iniciado en la ambulancia previo al PCI, logró una reducción significativa de eventos adversos cardiovasculares graves con un seguimiento a 3,8 años.

Rentoukas y colaboradores ${ }^{(6)}$ demostraron que el pos-CIR incrementó el porcentaje de pacientes que alcanzaron la renivelación completa del segmento ST y una reducción en la liberación de troponina I.

Crimi y colaboradores ${ }^{(7)}$, en un trabajo con 100 pacientes con STEMI, encontraron una reducción de $20 \%$ en la liberación de CK-MB medida como área bajo la curva (AUC CK-MB) y de $21 \%$ en el edema miocárdico medido con imágenes de resonancia magnética (IRM), con CIR realizado al comienzo de la reperfusión.

Munk y colaboradores ${ }^{(8)}$ analizaron el efecto del CIR en 242 pacientes con STEMI. El grupo al que se le realizó CIR (cuatro ciclos de 5 minutos de isquemia reperfusión en el miembro superior) previo a la realización de PCI, presentó una mejoría de la función ventricular izquierda, fundamentalmente en el grupo de alto riesgo, por presentar extenso territorio miocárdico afectado.

En el trabajo de White y colaboradores ${ }^{(9)}, 197$ pacientes con STEMI y flujo TIMI 0 fueron randomizados a un grupo de per-CIR (cuatro ciclos de 5 minutos de isquemia/reperfusión mediante insuflación-desinsuflación del manguito del esfingomanómetro en el miembro superior) o a un grupo control (aplicación del manguito durante 40 minutos sin insuflación) previo a PCI. El grupo con per-CIR redujo el TI en $27 \%$ en comparación con el grupo control. La liberación de troponina $\mathrm{T}$ fue menor en el grupo per-CIR, se redujo la extensión del edema miocárdico medido con IRM y aumentó el área de miocardio salvado en relación con el área de miocardio en riesgo.

Yellon y colaboradores, en el trabajo ERIC LYSIS(10) (que testeó el efecto del CIR en pacientes con STEMI que recibieron fibrinolíticos ), reclutaron 519 pacientes que fueron randomizados en dos grupos. El grupo CIR recibió cuatro ciclos de $5 \mathrm{mi}-$ nutos de isquemia/reperfusión en el miembro superior antes de la trombolisis. Se demostró una reducción de $17 \%$ en la liberación enzimática (CK-MB y troponinas) en el grupo CIR.

Gaspar y colaboradores(11) incluyeron 258 pacientes con STEMI a los que se randomizó a un grupo con CIR (tres ciclos de 5 minutos de isquemia-reperfusión mediante oclusión del miembro inferior previo al PCI) o a un grupo control. La mortalidad por causa cardiovascular y la hospitalización vinculada a insuficiencia cardíaca (IC) fueron menores en el grupo CIR en comparación con el grupo control (HR: 0,35; IC95\% 0,15-0,78). Lo mismo ocurrió con la necesidad del uso de diuréticos, inotrópicos y de balón de contrapulsación. Luego de 12 meses de seguimiento ecocardiográfico la recuperación de la fracción de eyección ventricular izquierda fue mayor en el grupo con CIR.

Pryds y colaboradores ${ }^{(12)}$, en un análisis post-hoc, determinaron que el CIR atenúa el deterioro miocárdico generado por el atraso en el PCI en pacientes con STEMI. En los pacientes donde el tiempo desde el inicio del dolor hasta la realización de un PCI fue mayor a 120 minutos, el grupo con CIR aumentó el porcentaje de miocardio salvado en comparación con el grupo control.

Sloth y colaboradores, también en un análisis post-hoc(13), encontraron que luego de un seguimiento de cuatro años el costo medio vinculado a la medicación fue menor en el grupo CIR, debido a menor ocurrencia de eventos cardio o cerebrovasculares, sugiriendo que el empleo de CIR en pacientes con STEMI y sometidos a PCI sería una estrategia costo-beneficio favorable. 
En los últimos años ha surgido un concepto denominado condicionamiento isquémico remoto crónico (crónico-CIR) por el que episodios repetidos de CIR mantenido durante semanas luego del infarto tendrían efecto beneficioso en la función ventricular y en la remodelación miocárdica. Trabajos como el de Tong, recientemente publicado ${ }^{(14)}$, en el cual se generó crónico-CIR a un grupo de pacientes hipertensos (tres ciclos de 5 minutos de isquemia-reperfusión en el miembro superior, diariamente durante 30 días) demostró mejoría de la función endotelial medida mediante el índice de reactividad hiperémica y el control de las cifras tensionales. En la actualidad se encuentran en desarrollo varios trabajos tendientes a estudiar el crónico-CIR aisladamente o asociado a pre y pos-CIR como cardioprotectores frente al daño por isquemia-reperfusión.

Existen estudios clínicos e incluso metaanálisis discordantes con la bibliografía analizada en cuanto al rol del CIR en la protección del miocardio frente a la injuria por isquemia-reperfusión. Algunos de ellos incluyeron pacientes diabéticos con neuropatía. En muchos de estos pacientes las fibras sensoriales $\mathrm{C}$ están dañadas, lo que explica la atenuación del CIR en su rol cardioprotector, ya que son de suma importancia como mediadoras del CIR. Por otro lado, la diabetes mellitus afecta señales intracelulares que son cruciales para generar cardioprotección. Por este motivo, creemos importante excluir a los pacientes diabéticos, principalmente con neuropatía diabética, en futuros trabajos.

Por otra parte, una atenuación del CIR ha sido vista cuando se usa propofol ${ }^{(15)}$. El uso de este agente en lugar de anestésicos volátiles ha sido frecuente en trabajos que no demostraron efectividad del CIR en su rol cardioprotector durante la cirugía cardíaca.

Varios ensayos incluyeron a pacientes con STEMI con infartos pequeños (coronaria derecha o circunfleja), quienes no se benefician tanto de las estrategias cardioprotectoras como los que tienen gran territorio comprometido. Los fármacos que recibía el paciente previo al STEMI también impactan en la cardioprotección generada por el CIR, como es el caso de las sulfonilureas, que pueden atenuar la respuesta cardioprotectora. Por su parte, la insulina, la metformina, algunas estatinas y opioides pueden ser por sí mismos agentes cardioprotectores, pudiendo enmascarar el beneficio adicional que confiere el CIR.

De la bibliografía consultada ${ }^{(4,11)}$ se desprende que hasta el momento no hay un protocolo de CIR estandarizado en cuanto a número de ciclos a realizar ni sobre la duración de los mismos. No está establecido el momento óptimo de iniciar el condiciona- miento en el per ni en el pos-CIR, así como tampoco la duración exacta del crónico-CIR. No hay estudios concluyentes que demuestren superioridad de los miembros superiores o inferiores como estímulo del condicionamiento.

Como conclusión, y en relación con el caso clínico presentado, el CIR debería ser aplicado. Este paciente se encuentra dentro del grupo que más se beneficiaría de las estrategias cardioprotectoras por tratarse de un paciente joven, que presenta un infarto agudo de miocardio con gran sector miocárdico comprometido y que será sometido a un PCI. En este grupo, el CIR ha demostrado ser una técnica sencilla de implementar, no invasiva, con relación costo-beneficio favorable y avalada por trabajos clínicos bien diseñados. En ellos se demostró que el CIR permitió reducir el tamaño del infarto, mejorar la funcionalidad ventricular izquierda, disminuir la hospitalización por IC y la mortalidad de causa cardiovascular, con menor incidencia de eventos cerebro y cardiovasculares graves en seguimiento a mediano plazo.

Futuros trabajos, como el CONDI2/ERIC-PPCI trial con 5.413 pacientes, cuyos resultados estarán disponibles este año, quizá puedan aportar una mayor información que nos permita establecer conductas sólidamente fundamentadas en este tan interesante y aún no laudado tema.

Marcelo Espiñeira, https://orcid.org/0000-0002-5131-6561

\section{Bibliografía}

1. Lloyd-Jones D, Adams R, Carnethon M, De Simone G, Ferguson T B, Flegal K, et al. Heart disease and stroke statistics - 2009 update: a report from the American Heart Association Statistics Committee and Stroke Statistics Subcommittee. Circulation. 2009;119(3): e21-181. doi: 10.1161/ CIRCULATIONAHA.108.191261. Fe de erratas: Circulation. 2011 Oct 18;124(16):e424.

2. Heusch G, Libby P, Gersh B, Yellon D, Bohm M, Lopaschuk G, et al. Cardiovascular remodelling in coronary artery disease and heart failure. Lancet 2014;383(9932):1933-43. doi: 10.1016/S0140-6736 (14)60107-0

3. Skyschally A, Gent S, Amanakis G, Schulte C, Kleinbongard P, Heusch G. Across-Species Transfer of Protection by Remote Ischemic Preconditioning With Species-Specific Myocardial Signal Transduction by Reperfusion Injury Salvage Kinase and Survival Activating Factor Enhancement Pathways. Circ Res. 2015;117(3):279-88. doi: 10.1161/ CIRCRESAHA.117.306878

4. Bøtker HE, Kharbanda R, Schmidt MR, Bøttcher M, Kaltoft AK, Terkelsen CJ, et al. Re- 
mote ischemic conditioning before hospital admission, as a complement to angioplasty, and effect on myocardial salvage in patients with acute myocardial infarction: a randomised trial. Lancet 2010;375 (9716): 727-34. doi: 10.1016/S0140-6736(09)62001-8

5. Sloth AD, Schmidt MR, Munk K, Kharbanda RK, Redington AN, Schmidt M, et al. Improved long-term clinical outcomes in patients with ST-elevation myocardial infarction undergoing remote ischemic conditioning as an adjunct to primary percutaneous coronary intervention. Eur Heart J. 2014; 35(3):168-75. doi: 10.1093/eurheartj/eht369

6. Rentoukas I, Giannopoulos G, Kaoukis A, Kossyvakis C, Raisakis K, Driva M, et al. Cardioprotective role of remote ischemic periconditioning in primary percutaneous coronary intervention: enhancement by opioid action. JACC Cardiovasc Interv. 2010;3(1):49-55. doi: 10.1016/j.jcin.2009. 10.015

7. Crimi G, Pica S, Raineri C, Bramucci E, De Ferrari GM, Klersy C, et al. Remote ischemic post-conditioning of the lower limb during primary percutaneous coronary intervention safely reduces enzymatic infarct size in anterior myocardial infarction: a randomized controlled trial. JACC Cardiovasc Interv. 2013;6(10):1055-63. doi: 10.1016/j.jcin.2013. 05.011

8. Munk K, Andersen NH, Schmidt MR, Nielsen SS, Terkelsen CJ, Sloth E, et al. Remote ischemic conditioning in patients with myocardial infarction treated with primary angioplasty: impact on left ventricular function assessed by comprehensive echocardiography and gated single-photon emission CT. Circ Cardiovasc Imaging 2010;3(6):656-62. doi: 10.1161/CIRCIMAGING.110.957340

9. White SK, Frohlich GM, Sado DM, Maestrini V, Fontana M, Treibel TA, et al. Remote ischemic conditioning reduces myocardial infarct size and edema in patients with ST-segment elevation myocar- dial infarction. JACC Cardiovasc Interv. 2015;8(1Pt B):178-88. doi: 10.1016/j.jcin.2014.05.015

10. Yellon DM, Ackbarkhan AK, Balgobin V, Bulluck H, Deelchand A, Dhuny MR, et al. Remote ischemic conditioning reduces myocardial infarct size in STEMI patients treated by thrombolysis. J Am Coll Cardiol.2015;65(25):2764-65. doi: 10.1016/j.jacc. 2015. 02.082

11. Gaspar A, Lourenco AP, Pereira MA, Azevedo P, Roncon-Albuquerque RJ, Marques J, et al. Randomized controlled trial of remote ischemic conditioning in ST-elevation myocardial infarction as adjuvant to primary angioplasty (RIC-STEMI). Basic Res Cardiol. 2018;113(3):14. doi: 10.1007/s00395018-0672-3

12. Pryds K, Terkelsen CJ, Sloth AD, Munk K, Nielsen SS, Schmidt MR, et al. Remote ischemic conditioning and healthcare system delay in patients with ST-segment elevation myocardial infarction. Heart 2016;102(13):1023-28. doi: 10.1136/heartjnl2015-308980

13. Sloth AD, Schmidt MR, Munk K, Schmidt M, Pedersen L, Sørensen HT, et al. Cost-effectiveness of remote ischemic conditioning as an adjunct to primary percutaneous coronary intervention in patients with ST-elevation myocardial infarction. Eur Heart Journal Acute Cardiovasc Care 2016:1-10. doi: $10.1177 / 2048872615626657$

14. Tong XZ, Cui WF, Li Y, Su C, Shao YJ, Liang $\boldsymbol{J W}$, et al. Chronic remote ischemic preconditioning-induced increase of circulating hSDF- $1 \alpha$ level and its relation with reduction of blood pressure and protection endothelial function in hypertension. J Hum Hypertens.2019 Jan 10. doi.org/10.1038/ s41371- 0180151-1

15. Bautin AE, Galagudza MM, Datsenko S V, Tashkhanov DM, Marichev AO, Bakanov AI, et al. Effects of remote ischemic preconditioning on perioperative period in elective aortic valve replacement. Anesteziol Reanimato.2014:11-17. Ruso. 


\section{No es esperable que el condicionamiento isquémico remoto aporte beneficios significativos en el caso que se presenta}

\section{Dra. Luciana Jubany Manfrini}

\section{Análisis del caso clínico}

Los datos de mayor importancia en este caso son:

- Hombre joven, portador de importantes factores de riesgo cardiovascular.

- Cursando un infarto agudo de miocardio con elevación del segmento ST (IAMCEST) de 30 minutos de evolución al momento del arribo de la unidad de emergencia móvil.

- Residente en Montevideo.

- Arribo a sala de hemodinamia a los 55 minutos de iniciados los síntomas.

- Angioplastia primaria efectiva (flujo TIMI 3) sobre oclusión trombótica de arteria descendente anterior (ADA).

\section{Discusión}

El IAMCEST es una de las principales causas de mortalidad y morbilidad en nuestro país. El tamaño del infarto es un importante determinante en la evolución de estos pacientes, por lo que las medidas tendientes a su reducción constituyen un pilar terapéutico central. La medida de mayor relevancia, como lo indican las guías actuales, es la reperfusión precoz, ya sea mediante el uso de fibrinolíticos o la angioplastia primaria, sabiendo que esta última es el tratamiento de elección si se puede realizar rápidamente (120 minutos desde el diagnóstico $)^{(1)}$.

De todas formas, aunque la reperfusión se realice en forma precoz, el riesgo continúa siendo significativo, por lo cual se han explorado nuevas estrategias con el fin de reducir el tamaño del infarto.

Una línea de trabajo se fundamenta en que la inducción de episodios breves no letales de isquemia y reperfusión sobre el corazón antes, o incluso después de un episodio de isquemia miocárdica prolongada, podría tener la capacidad de reducir la lesión miocárdica que ésta genera. Surgió luego la idea de que podrían lograrse niveles similares de cardioprotección aplicando los episodios breves de isquemia y reperfusión a un órgano o tejido alejado, evitando así la necesidad de "acondicionar" el corazón directamente, siendo este mecanismo denominado con- dicionamiento isquémico remoto. Esta mayor tolerancia a los efectos de la lesión aguda por isquemia-reperfusión (condicionamiento isquémico cardíaco) ha sido investigada mediante la aplicación de varias estrategias mecánicas y farmacológicas diferentes $^{(2)}$.

Una forma de condicionamiento remoto es generar isquemia intermitente en un miembro luego del desarrollo de los síntomas y previo a la reperfusión (percondicionamiento), pudiendo ser iniciado durante el traslado del paciente ${ }^{(3)}$.

Analizando la evidencia disponible, actualmente surge la duda sobre la real eficacia de este procedimiento. En 2010, Botker publicó un estudio randomizado comparando dos poblaciones cursando IAMCEST. Ambas recibieron angioplastia primaria, con o sin condicionamiento remoto, mediante cuatro ciclos de 5 minutos de inflado y 5 minutos de desinflado de un manguito de presión a nivel de un miembro superior. Su objetivo primario fue el índice de miocardio salvado a 30 días del evento medido por SPECT (Single Photon Emission Computed Tomography). Los objetivos secundarios fueron: tamaño final del infarto, concentración de troponinas, muerte, reinfarto, reingreso a 30 días por falla cardíaca, fracción de eyección del ventrículo izquierdo (FEVI) y clase funcional según la New York Heart Association a 30 días. De los resultados, se destaca que el porcentaje de músculo salvado fue mayor en el grupo de la intervención, con menor tamaño del infarto pero con diferencia no significativa. A 30 días, la FEVI no fue significativamente diferente entre los dos grupos y no hubo diferencias en cuanto a eventos mayores. En conclusión, el condicionamiento remoto aumenta el miocardio salvado y es un procedimiento seguro y de bajo costo, lo que lo hace atractivo para ser evaluado en ensayos clínicos de mayor escala ${ }^{(3)}$.

Heusch plantea, en un artículo de revisión sobre el tema, que el traslado de las estrategias de cardioprotección de lo experimental a la clínica ha sido en cierta manera desalentador, debido a los diseños de los estudios y a la participación de factores confun-

Instituto Nacional de Cirugía Cardíaca. Montevideo, Uruguay.

Correspondencia: Dra. Luciana Jubany. Correo electrónico: luciana.jubany@gmail.com

La autora declara no tener conflictos de intereses.

Recibido May 20, 2019; aceptado Jun 11, 2019. 
didores en los pacientes, tales como comorbilidades, tratamientos farmacológicos previos, duración del dolor y tiempo hasta la intervención. El autor considera apropiado centrar las investigaciones en el condicionamiento remoto, para lo cual habría que optimizar los protocolos (número y duración de los ciclos) y comprender mejor los mecanismos y señales involucrados de forma de disminuir el efecto de los factores confundidores ${ }^{(4)}$.

Un metaanálisis realizado en 2016 por Elbadawi, que incluye ensayos clínicos que evalúan el condicionamiento remoto, utiliza como punto final primario el tamaño del infarto valorado por la concentración de biomarcadores (troponinas y CPK-MB), demostrando una reducción por este método en los pacientes tratados. En cuanto al punto final compuesto por infarto de miocardio, mortalidad por todas las causas, mortalidad cardiovascular y accidente cerebrovascular isquémico, uno de los puntos finales secundarios, los pacientes tratados tuvieron mejores resultados, pero los estudios incluidos no tenían el suficiente poder estadístico para este tipo de análisis ${ }^{(5)}$.

En el caso que nos ocupa, el paciente consulta en forma muy precoz, apenas 30 minutos después de iniciados los síntomas, y el traslado se realiza en forma rápida, con una duración de 25 minutos, por lo que creemos que la mejor estrategia es realizar los tratamientos que actualmente están avalados por las guías de la práctica clínica, efectuando la angioplastia primaria del vaso culpable con el tratamiento adyuvante en base a antiagregantes y anticoagulantes con eficacia demostrada. De hecho, estudios realizados plantean el protocolo prehospitalario cuando el tiempo de traslado se prevé que va a exceder los 20 a 30 minutos $^{(2)}$, y el mayor beneficio se da en pacientes con 3 a 8 horas de evolución de los síntomas previo a la reperfusión ${ }^{(4)}$.

No obstante, actualmente está en curso un ensayo clínico randomizado, Effect of Remote Ischaemic Conditioning on Clinical Outcomes in ST-segment Elevation Myocardial Infarction in Patients Undergoing Primary Percutaneous Coronary Intervention (CONDI2/ERIC-PPCI), con 5.413 pacientes enrolados, del que se espera tener los resultados en diciembre de este año, teniendo como end points pri- marios mortalidad cardíaca y hospitalización por falla cardíaca. Quizá este nuevo estudio pueda cambiar la conducta al respecto en estos $\operatorname{casos}^{(6)}$.

Luciana Jubany Manfrini,

https://orcid.org/0000-0003-3347-8492

\section{Bibliografía}

1. Ibáñez B, James S, Agewall S, Antunes MJ, Bucciarelli-Ducci Ch, Bueno H, et al. 2017 ESC Guidelines for the management of acute myocardial infarction in patients presenting with ST-segment elevation: the task force for the management of acute myocardial infarction in patients presenting with ST-segment elevation of the European Society of Cardiology (ESC). Eur Heart J. 2018;39 (2): 119-77. doi: 10.1093/eurheartj/ehx393

2. Hausenloy DJ, Yellon DM. Preconditioning and postconditioning: Underlying mechanisms and clinical application. Atherosclerosis 2009;204(2):334-41. doi: 10.1016/j.atherosclerosis.2008.10.029

3. Bøtker HE, Kharbanda R, Schmidt MR, Bøttcher M, Kaltoft AK, Terkelsen CJ, et al. Remote ischemic conditioning before hospital admission, as a complement to angioplasty, and effect on myocardial salvage in patients with acute myocardial infarction: a randomised trial. Lancet 2010; 375(2916):72734. doi: 10.1016/S0140-6736(09)62001-8

4. Heusch G, Bøtker HE, Przyklenk K, Redington A, Yellon D. Remote Ischemic Conditioning. J Am Coll Cardiol. 2015;65(2):177- 95. doi: 10.1016/j.jacc. 2014.10.031.

5. Elbadawi A, Ha LD, Abuzaid AS, Crimi G, Azzouz MS. Meta-Analysis of randomized trials on remote ischemic conditioning during primary percutaneous coronary intervention in patients with ST-segment elevation myocardial infarction. Am J Cardiol. 2016;119(6):832-8. doi: 10.1016/j.amjcard. 2016.11

6. Hausenloy D, Botker HE. Effect of remote ischemic conditioning on clinical outcomes in STEMI patients undergoing PPCI (CONDI2/ERIC-PPCI) [Internet]. Bethesda: ClinicalTrial.gov; 2018 [Consulta 30 May 2019]. Disponible en: https://clinicaltrials.gov/ct2/show/NCT02342522 


\section{Respuesta a la Dra. Luciana Jubany Manfrini}

Es indiscutible el papel fundamental de la angioplastia primaria o la reperfusión con fibrinolíticos, cuando la primera no se encuentra disponible, en el tratamiento de pacientes con IAM con supradesnivel del ST. Sin embargo, la cardiopatía isquémica aguda continúa siendo la principal causa de muerte en el mundo desarrollado. Está demostrado que la reperfusión en sí misma supone un daño al miocardio, contribuyendo en un 40\%-50\% en el tamaño del infarto (TI), por lo que es imperativo generar estrategias de cardioprotección. Es aquí donde el condicionamiento isquémico remoto (CIR) cumpliría un rol importante como tratamiento adyuvante a las estrategias de reperfusión (nunca como sustituto de las mismas).

Los trabajos menos alentadores en el desarrollo de esta técnica reconocen mayor porcentaje de músculo miocárdico salvado y menor TI en los pacientes a los que se les aplicó el CIR, reconociendo problemas en el diseño de los estudios y factores confundidores para demostrar los otros beneficios clínicos. Sabemos que hay trabajos (referenciados en la bibliografía) que demuestran además benefi- cios en la funcionalidad ventricular izquierda, disminución de la hospitalización por insuficiencia cardíaca y menor incidencia de eventos cerebro y cardiovasculares graves. $\mathrm{Al}$ ser una técnica sencilla de implementar, no invasiva, con relación costo-beneficio favorable, en la cual no hay descritas contraindicaciones ni efectos adversos graves adjudicables al procedimiento y a que no retrasaría el comienzo de las medidas de reperfusión por poder aplicarlo durante el traslado en ambulancia del paciente o mientras se prepara la sala de hemodinamia; consideramos que sería beneficioso el empleo del CIR. Aún más en este paciente que por las características que presenta (enumeradas en el trabajo) se encuentra dentro del grupo que más se beneficia de esta estrategia cardioprotectora.

Coincidimos en que futuros trabajos, como el CONDI2/ERIC-PPCI trial, cuyos resultados tendremos disponibles este año, y otros en los cuales se unifique un protocolo de CIR estandarizado, son necesarios para elaborar pautas que protocolicen futuras conductas.

Dr. Marcelo Espiñeira

co randomizado CONDI2/ERIC-PPCI va a aportar mayor información en cuanto a los beneficios en mortalidad y hospitalización por falla cardíaca y va a permitir protocolizar el uso del condicionamiento isquémico remoto como medida complementaria a la reperfusión precoz.

Dra. Luciana Jubany Manfrini 